\title{
Dynamic immunity gene and IncRNA regulatory cooperative pairs revealed prognostic signatures in the development of colon cancer
}

\section{Chun Jing Wang}

Department of General surgery, the Second Affiliated Hospital of Harbin Medical University, Heilongjiang, China

\section{Liu Ping You}

Department of General surgery, the Second Affiliated Hospital of Harbin Medical University, Heilongjiang, China

\section{Fujing Wang ( $\nabla$ wangfujing-hyd@163.com )}

Second Affiliated Hospital of Harbin Medical University

Hao Cui

Department of Ultrasound, the Second Affiliated Hospital of Harbin Medical University, Heilongjiang, China

Yu Zhou Hu

Department of General surgery, the Second Affiliated Hospital of Harbin Medical University, Heilongjiang, China

\section{Sheng Long Li}

Department of General surgery, the Second Affiliated Hospital of Harbin Medical University, Heilongjiang, China

\section{Research}

Keywords: Immunity; Long non-coding RNA; Cancer progression; Colon cancer; Prognosis

Posted Date: August 4th, 2020

DOI: https://doi.org/10.21203/rs.3.rs-46727/v1

License: (c) (i) This work is licensed under a Creative Commons Attribution 4.0 International License. Read Full License 


\section{Abstract}

Background: The pathological development of colon cancer is a complex progression that depends on multiple alterations of coding and non-coding genes. Colon cancer are challenged by the immune system and acquire features to evade its surveillance during the whole process of occurrence and development. Therefore, it is important to capture the immune regulatory events during the progression of colon cancer development and to identify reliable markers for predicting clinical outcomes in patients.

Methods: Here, a standardized computational procedures was developed to evaluate immune cell populations-associated gene (immuneCPa gene)-IncRNA relationships in diverse stages (I, II, III and IV) of colon cancer based on genes and IncRNAs expression. Stage-specific immuneCPa genes and IncRNAs were identified in each colon cancer stage.

Results: Dynamic stage-specific immuneCPa gene-IncRNA regulatory networks were constructed and characterized in colon cancer development. A immuneCPa gene-IncRNA activity profile across different stages revealed that IncRNAs were highly stage-selective in regulating immuneCPa genes in colon cancer. Specify scores of immune indicated that diverse kinds of immune cells were temporally-specific in colon cancer. NK CD56bright cell showed strongest stage common features in colon cancer. Further survival analysis indicated that some stage specific immuneCPa gene-IncRNA relationships may have the potential for predicting colon cancer prognosis.

Conclusions: Collectively, our study leads to a novel starting point for future functional explorations, the identification of immune-related biomarkers, and IncRNA-based targeted therapy for colon cancer.

\section{Background}

Colon cancer represents the third most frequent cancer in the world, with 147,950 novel cases worldwide in 2020 [1]. $30 \%$ of patients would occur recurrence when there is nodal involvement (stage III) due to micrometastatic spreading although the patients are diagnosed at a localized stage [2]. Colon cancer is a potentially preventable disease and early-stage colon cancer is usually curable [3]. Although the survival of colon cancer patients improve follow the combination of different therapeutic approaches including surgery, endoscopic ablation, radiation, and chemotherapy, individualization of patients varies greatly. Especially, patients with advanced cancer show worse prognosis. Therefore, it is important to understand the mechanisms regulating the progression of colon cancer and to identify efficacious therapeutic targets and prognostic/predictive biomarkers for predicting clinical outcomes in patients.

The past years have witnessed promising clinical feedback for anti-cancer immunotherapies. Immune cells contribute to invasion by secreting a cornucopia of inflammatory factors that promote epithelial-tomesenchymal transition and remodeling of the stroma [4]. Previous study reports that immunoscore is a robust and validated clinical assay leveraging immune scoring to predict recurrence risk of colon cancer patient [5]. Immune-related genes play regulatory roles in the immune system and are involved in the initiation and progression of colon cancer [6]. Exploring the roles of immune in diverse stages of colon 
cancer could provide assistance for immunotherapy of colon cancer. However, most current researches only focus on immune-related coding genes in cancers.

In recent years, emerging evidence has shown the importance of long non-coding RNAs (IncRNAs) as a new regulator of many physical or pathological processes $[7,8]$. IncRNAs also play essential roles in colon cancer. For example, LINC00662 overexpression promoted the occurrence and development of colon cancer [9]. LINC00460 might function as an oncogenic IncRNA in colon cancer development and could be explored as a potential biomarker and therapeutic target for colon cancer. The functions of IncRNA in immune response for cancers are also identified. IncRNA could control the modulation of immune checkpoint molecules in cancer [10]. For example, IncRNA NKILA promotes tumor immune evasion by sensitizing T cells to activation-induced cell death [11]. Interference IncRNA SNHG1 could inhibit the differentiation of Treg cells by promoting miR-448 expression, thereby impeding the immune escape of breast cancer [12]. These studies have significantly enhanced our understanding of the IncRNA mechanisms in immune and underlying disease progression. Unfortunately, there has only been limited work studying the dynamic immune-related gene-IncRNA interactions involved in colon cancer development.

To address this issue, the present study designed an integrated computational approach to identify immuneCPa gene-IncRNA pairs in diverse stages of colon cancer based on genes and IncRNAs expression. Stage-specific immuneCPa genes and IncRNAs were extracted in each colon cancer stage. Dynamic stage-specific immuneCPa gene-IncRNA regulatory networks were constructed and characterized in colon cancer development. A immuneCPa gene-IncRNA activity profile across different stages revealed that IncRNAs were highly stage-selective in regulating immuneCPa genes in colon cancer. Specify scores of immune indicated that diverse kinds of immune cells were temporally-specific in colon cancer. NK CD56bright cell showed strongest stage common features in colon cancer. Further survival analysis indicated that some stage specific immuneCPa gene-IncRNA relationships may have the potential for predicting prognosis in specific stage. In summary, our systematic analysis not only sheds new light on dynamic regulatory mechanisms of immuneCPa gene-IncRNA interactions, but may also help in colon cancer prognosis stratification and discovery of therapeutic targets.

\section{Materials And Methods}

\section{Obtain of high-throughput expression profiles of IncRNAs and genes for colon cancer}

Gene and IncRNA expression profiles (Level 2) were obtained from The Cancer Genome Atlas (TCGA, https://www.cancer.gov/about-nci/organization/ccg/research/structural-genomics/tcga). 464colon cancer tissues and 101 tumor adjacent normal control tissues were got and clinical follow-up information including cancer stages and survival data were retained for further analysis. Lastly, 90, 226, 160 and 81 stage I, II, II and IV colon patients samples were obtained. The genes and IncRNAs which their expression values were 0 in all the samples would be removed. All expression values had been transformed by $\log _{2}$ to satisfy normal distribution. 


\section{Collecting multiple kinds of immune cell populations-associated genes}

17 selected immune cell populations including B cells, Eosinophils, Macrophages, Mast cells, NK CD56bright cells, NK CD56dim cells, Neutrophils, T helper cells, Tcm cells, Tem cells, Tfh cells, aDC, iDC, Activated CD8 T cell, Gamma delta T cell, Regulatory T cell and Cytotoxic cells were selected [13]. Multiple kinds of immune cell populations-associated genes (immuneCPa genes) were also obtained from previous study $[14,15]$. All the ImmuneCPa genes were retained for further analysis.

\section{Identifying colon cancer stage-specific immuneCPa genes and IncRNAs based on expression profiles}

In order to identify colon cancer stage-specific immuneCPa genes and IncRNAs, t-test was used to perform differential expression analysis between expression profiles of colon cancer and normal control samples in four diverse stages. The significant immuneCPa genes $(P<0.05)$ were considered as colon cancer stage-specific immuneCPa genes. The significant IncRNAs $(P<0.01)$ were considered as colon cancer stage-specific IncRNAs. Only the colon cancer stage-specific immuneCPa genes and IncRNAs were used for follow analyses.

\section{Construction of stage-specific immuneCPa gene-IncRNA regulatory networks in colon cancer development}

For each kind of immune cell populations, Pearson correlation coefficients (PCCs) were calculated for each colon cancer stage-specific immuneCPa genes and IncRNAs in colon cancer patients with diverse stages, respectively. The PCCs and $P$ values were obtained for each stage-specific immuneCPa geneIncRNA pair. Only the stage-specific immuneCPa gene and IncRNA pairs with absolute values which more than 0.5 were used for follow analyses and network construction. The stage-specific immuneCPa geneIncRNA regulatory networks were constructed by Cytoscape 3.3.0 (https://cytoscape.org/). Degree analysis was also performed by Cytoscape 3.3.0.

\section{The specificity score of stage-specific immuneCPa gene-IncRNA pairs for colon cancer}

The specificity of each stage-specific immuneCPa gene-IncRNA pair across different stages of colon cancer was determined by the specificity score as: (see Equation 1 in the Supplemental Files)

Where $\mathrm{N}$ is the number of colon cancer stages and $\mathrm{PCC}_{\mathrm{i}}$ is a component normalized to the maximum value of the PCC value. Specify score was used to evaluate stage specific for stage-specific immuneCPa gene-IncRNA pair in colon cancer.

\section{Survival analyses for stage-specific immuneCPa gene-IncRNA pairs in colon cancer}

In order to evaluate the prognosis about stage-specific immuneCPa gene-IncRNA pairs in colon cancer, we performed survival analysis for these four stage groups. The two groups were used as constructing and validating model, respectively. We used the regression coefficient of each immuneCPa gene and IncRNA in the stage-specific immuneCPa gene-IncRNA pair related to patient survival based on gene and IncRNA 
expression data to verify if these pairs were associated with survival. First, the colon cancer patients in each stage were randomly divided into two groups and the samples in these two groups are independent. Second, a multivariate Cox regression model was applied to each immuneCPa gene and its interacted IncRNAs in diverse colon cancer stage to obtain a standardized Cox regression coefficient for the first group. Age, cancer stage and sex were also considered as confounders in this process. Third, a risk score formula was constructed based on expression values of each immuneCPa gene and its interacted IncRNAs for the held-out group weighed by their estimated regression coefficients, following the above multivariate Cox regression analysis. Fourth, median of the risk score was used as the threshold value to divide the colon cancer patients into high-risk and low-risk groups. Finally, Kaplan-Meier (K-M) survival analysis was used for the diverse risk groups. Statistical significance assessed using the log-rank test. All analyses were performed within the R 2.6.6 framework.

\section{Results}

\section{Some stage-specific immuneCPa genes and IncRNAs were identified in colon cancer}

We first identified colon cancer-specific 17 kinds of immuneCPa genes for colon cancer in all stages. More than 70\% of the 15 kinds of immuneCPa genes were differentially expressed (Figure 1A). 96.97\% B cells associated genes were differentially expressed in all the colon cancer patients. The result indicated that immuneCPa genes play important roles in colon cancer. Next, stage-specific immuneCPa genes were identified in diverse colon cancer stages. In each kind of immuneCPa gene, percent of differential expressed immuneCPa genes showed similar pattern (Figure 1B). Most stage-specific immuneCPa genes were presented in patients with stage II colon cancer which is a critical stage of cancer progression. The result suggested that stage II was active stage of immune system to fight cancer cells. Diverse stage also shared some common immuneCPa genes (Figure 1C). For example, four stages had 14, 9 and 10 common immuneCPa genes in B cells, Gamma delta T cell and Eosinophils. We also extracted differential expressed IncRNAs (61\%) for all colon cancer patients (Figure 1D). Similar to immuneCPa genes, there were most differential expressed IncRNAs in stage II of colon cancer. Four diverse stages shared more common differential expressed IncRNAs compared with immuneCPa genes (Figure 1E). We inferred some key IncRNAs maybe functions essentially in specific colon cancer stage.

\section{Identification of stage-specific immuneCPa gene-IncRNA regulatory pairs in colon cancer development}

Two major factors including interactions and similar expression to extract stage-specific immuneCPa gene-IncRNA regulatory pairs. Experimentally verified gene-IncRNA interaction were considered as candidate regulatory paris. Then considering that interactions do not directly imply their actual regulation of immuneCPa gene-IncRNA pairs in certain conditions, exploring gene regulation of IncRNAs through coexpression analysis can offer useful information to identify active immuneCPa gene-IncRNA relationships in different colon cancer stages. PCCs were calculated for each potential immuneCPa geneIncRNA pair based on their expression profiles at different stages (Figure 2A). Stage II and III showed most similar distribution of PCCs for gene-IncRNA pairs. Most PCCs of gene-IncRNA pairs were 
concentrated between 0.5 and 1 or -0.5 and -1 (Figure 2B). It indicated that most gene-IncRNA pairs showed strong co-expressed level. The numbers of co-expressed gene-IncRNA pairs were diverse in different kinds of immune cells (Figure 2C). For example, there were most co-expressed gene-IncRNA pairs in Tem and Tfh cells. In addition, most co-expressed gene-IncRNA pairs were present in stage III for almost kinds of immune cells (Figure 2D). The different sizes of these stage-specific immuneCPa geneIncRNA pairs indicate the heterogeneity of immune associated genes and IncRNAs in the development of colon cancer.

\section{Construction of dynamic stage-specific immuneCPa gene-IncRNA regulatory networks in colon cancer development}

We used a $\mathrm{P}<0.05$ as the thresholds to identify a link between immuneCPa genes and IncRNAs in the regulatory networks. There were $91,962,106,834,165,589$ and 118,431 immuneCPa gene and IncRNA pairs in stage I, II, III, IV (Figure 3A). We extracted absolute values of PCCs $>0.9$ as the thresholds to construct more tighter and closer stage-specific immuneCPa gene-IncRNA regulatory networks in colon cancer development (Figure 3B). Specifically, 19,929 edges between 408 immuneCPa genes and 3,768 IncRNAs, 75,259 edges between 408 immuneCPa genes and 4,920 IncRNAs, 52,635 edges between 409 immuneCPa genes and 3,983 IncRNAs and 118,356 edges between 407 TFs and 4,389 IncRNAs were constructed for stages I, II, III and IV colon cancer patients, respectively. In these stage-specific immuneCPa gene-IncRNA regulatory networks, there were some key hub nodes such as immuneCPa gene DCSTAMP, KIR3DL3 and SND1-IT1 could interacted with more than 400 IncRNAs. In these four stagespecific immuneCPa gene-IncRNA regulatory networks, immuneCPa gene had bigger degree than IncRNAs (Figure 3 C). The degree analysis indicated that a common immuneCPa gene could be regulated by diverse and a number of IncRNAs in colon cancer development. IncRNAs maybe play specific regulatory roles for immuneCPa genes in diverse stages of colon cancer.

\section{The dynamic activity profiles of immuneCPa gene-IncRNA regulatory pairs}

Although stage-specific networks share common topological properties, the immuneCPa gene-IncRNA regulatory interactions may change in different stages of colon cancer. To evaluate the proportion of common and specific immuneCPa gene-IncRNA regulatory interactions during colon cancer progression, we explored the overlaps of immuneCPa gene-IncRNA regulatory relationships among four stage-specific networks (Figure 4A). Most immuneCPa gene-IncRNA regulatory relationships are stage specific. Stage III had maximum number of immuneCPa gene-IncRNA regulatory relationships (Figure 4B). We inferred that stage III is a key stage which IncRNA participated in immune regulation for colon cancer. For all the immuneCPa gene-IncRNA regulatory relationships, 378,658 immuneCPa gene-IncRNA regulatory relationships were only present in one stage. The four stages only shared 249 common immuneCPa gene-IncRNA regulatory relationships, indicating that relationships were temporally-specific in colon cancer (Figure $4 \mathrm{C}$ ). The immuneCPa gene-IncRNA regulatory relationships were making dynamic changes in colon cancer development (Figure 4D). To provide an overview of all possible immuneCPa geneIncRNA relationships and their dynamic regulatory status, we built an activity profile for immuneCPa 
gene-IncRNA relationships across different stages of colon cancer. Activity score is the standardized value of PPCs. Based on the activity scores, these immuneCPa gene-IncRNA relationships were grouped by the K-means clustering method (Figure 4E). Different groups of immuneCPa gene-IncRNA relationships were apparently activated at one or more stages. The patterns of immuneCPa gene-IncRNA relationships were diverse in different groups. These groups maybe could as stage-specific biomarkers for colon cancer.

\section{Specificity score evaluates the specificity of immuneCPa gene-IncRNA relationships in colon cancer development}

Specificity score was designed to evaluate the specificity of immuneCPa gene-IncRNA relationships in colon cancer development. Most immuneCPa gene-IncRNA relationships showed lower specificity scores and indicated stage specific for these relationships in colon cancer (Figure 5A). In order to extract more stage-specific immuneCPa genes, we used mean specificity scores of all immuneCPa gene-IncRNA relationships to represent the specify of each immuneCPa gene. These stage-specific immuneCPa genes could interact with diverse numbers of IncRNAs in colon cancer (Figure 5B). The mean specificity scores for each kind of immune cells were also obtained. Mast and cytotoxic cells showed the strongest stage specificity in colon cancer (Figure 5C). Specificity scores of most immuneCPa genes in these two kinds of immune cells concentrated between 0.06 and 0.08 (Figure 5D). NK CD 56bright cell showed strongest stage common features in colon cancer and indicated that it maybe participate in all immune process for colon cancer development. This results also revealed that diverse kinds of immune cells were temporallyspecific in colon cancer.

\section{Some immuneCPa gene-IncRNA relationships in colon cancer development has specific prognostic potential}

To evaluate the potential value of immuneCPa gene-IncRNA pairs as prognostic biomarkers in colon cancer with diverse stages, we created a risk-score formula according to the expression of each immuneCPa gene and its corresponding IncRNAs to generate OS (overall survival) prediction (see the Material and Methods section). We used median risk score as the cut-off point to test the survival of the diverse stage colon cancer patients into high-risk or low-risk groups. These three immuneCPa geneIncRNA clusters were associated with Macrophages, iDC and Neutrophils cell populations (Figure 6A). Two stage II and one stage III specific immuneCPa gene-IncRNA clusters were significantly associated with survival, and they could serve as prognostic biomarkers (Figure 6B).

\section{Discussion}

In the past few years, a number of studies have successfully characterized the roles of IncRNAs based on the general assumption that IncRNAs are key regulators of biological processes in many kinds of cancers. Although many studies have been carried out to characterize the complex and multiple functions of IncRNAs including cell growth [16], apoptosis [17], autophagy, epithelial mesenchymal transformation [18] and so on, little is known about the roles of IncRNAs in immune regulations for colon cancer. 
Preliminary studies have shown that IncRNAs play important roles in the immune system and had became the focus of immunology [19]. Thus, it is urgent to comprehensive characterize the functions and mechanisms of IncRNAs in immune regulation for cancers. In present study, we used immune related genes as a bridge to identifying immune related IncRNAs in colon cancer.

The pathological development of colon cancer is a complex progression that depends on multiple alterations of coding and non-coding genes. Although our understanding of colon cancer has increased, the precise regulatory mechanisms about immune regulations underlying this complex disease are still not fully known. Therefore, it is important to uncover the immune regulating events during progression of colon cancer and identify reliable markers for predicting the clinical outcome of patients. Specially, exploring regulatory mechanisms of IncRNAs in diverse stage of colon cancer could help us understand the dynamic changes of the immune system in different stages of colon cancer. The present study indicated that stage II was a key immune regulatory stage which there were most dysregulatory immuneCPa genes in colon cancer. Most immuneCPa gene-IncRNA relationships were present in stage III which demonstrated this stage was a critical period for IncRNA to participate in regulation. In short, stage II and III were crucial periods of immunotherapy for colon cancer.

To evaluate if immuneCPa gene-IncRNA pairs could become as stage-specific prognostic biomarkers in colon cancer, we constructed risk scores to identify survival-related immuneCPa gene-IncRNA pairs. In this process, we divided each colon cancer stage patients to two independent sets.

One dataset was used for Cox regression analysis and another held-out dataset was applied for validating the model. This method could help avoiding overfitting. These survival-related immuneCPa gene-IncRNA pairs could become as potential stage-specific prognostic biomarkers for colon cancer.

\section{Conclusion}

Collectively, the present study developed a standardized computational procedures to evaluate immuneCPa gene-IncRNA relationships in diverse stages of colon cancer based on genes and IncRNAs expression. Stage-specific immuneCPa genes and IncRNAs were identified in each colon cancer stage. Dynamic stage-specific immuneCPa gene-IncRNA regulatory networks were constructed and analyzed in colon cancer development. A immuneCPa gene-IncRNA activity profile across different stages revealed that IncRNAs were highly stage-selective in regulating immuneCPa genes in colon cancer. Collectively, our study leads to a novel starting point for future functional explorations, the identification of immunerelated biomarkers, and IncRNA-based targeted therapy for colon cancer.

\section{Abbreviations}

immuneCPa gene: immune cell populations-associated gene; IncRNA: long non-coding RNA; TCGA: The Cancer Genome Atlas; PCC: Pearson correlation coefficients. 


\section{Declarations}

\section{Funding}

The key Project of Harbin Science and Technology Bureau 2017AB9BS038. The Project of Tianqing Stem Cell Co., Ltd.

\section{Disclosure of interest}

The authors declare that they have no competing interest.

Acknowledgments

Not applicable.

Availability of data and material

The data that support the findings of this study are available from the corresponding author upon reasonable request.

\section{Ethics approval and consent to participate}

Not applicable.

\section{Patient consent for publication}

Not applicable.

\section{Competing interests}

The authors declare that they have no competing interests.

\section{Consent for publication}

Not applicable.

\section{Authors' contributions}

WFJ conceived and designed the experiments, WCJ, YLP, and CH analyzed the data, HYZ and LSL validated the work, and WCJ wrote the manuscript.

\section{References}

1. Siegel RL, Miller KD, Jemal A: Cancer statistics, 2020. CA Cancer J Clin 2020, 70:7-30.

2. Taieb J, Andre T, Auclin E: Refining adjuvant therapy for non-metastatic colon cancer, new standards and perspectives. Cancer Treat Rev 2019, 75:1-11. 
3. De Robertis M, Poeta ML, Signori E, Fazio VM: Current understanding and clinical utility of miRNAs regulation of colon cancer stem cells. Semin Cancer Biol 2018, 53:232-247.

4. El-Kenawi A, Hanggi K, Ruffell B: The Immune Microenvironment and Cancer Metastasis. Cold Spring Harb Perspect Med 2020, 10.

5. Marliot F, Chen X, Kirilovsky A, Sbarrato T, El Sissy C, Batista L, Van den Eynde M, Haicheur-Adjouri N, Anitei MG, Musina AM, et al: Analytical validation of the Immunoscore and its associated prognostic value in patients with colon cancer. $J$ Immunother Cancer 2020, 8.

6. Zhang H, Qin C, Gan H, Guo X, Zhang L: Construction of an Immunogenomic Risk Score for Prognostication in Colon Cancer. Front Genet 2020, 11:499.

7. DiStefano JK: The Emerging Role of Long Noncoding RNAs in Human Disease. Methods Mol Biol 2018, 1706:91-110.

8. Gibb EA, Brown CJ, Lam WL: The functional role of long non-coding RNA in human carcinomas. Mol Cancer 2011, 10:38.

9. Cheng B, Rong A, Zhou Q, Li W: LncRNA LINC00662 promotes colon cancer tumor growth and metastasis by competitively binding with miR-340-5p to regulate CLDN8/IL22 co-expression and activating ERK signaling pathway. J Exp Clin Cancer Res 2020, 39:5.

10. Xu S, Wang Q, Kang Y, Liu J, Yin Y, Liu L, Wu H, Li S, Sui S, Shen M, et al: Long Noncoding RNAs Control the Modulation of Immune Checkpoint Molecules in Cancer. Cancer Immunol Res 2020, 8:937-951.

11. Huang D, Chen J, Yang L, Ouyang Q, Li J, Lao L, Zhao J, Liu J, Lu Y, Xing Y, et al: NKILA IncRNA promotes tumor immune evasion by sensitizing T cells to activation-induced cell death. Nat Immunol 2018, 19:1112-1125.

12. Pei $X$, Wang $X$, Li H: LncRNA SNHG1 regulates the differentiation of Treg cells and affects the immune escape of breast cancer via regulating miR-448/IDO. Int J Biol Macromol 2018, 118:24-30.

13. Tamborero D, Rubio-Perez C, Muinos F, Sabarinathan R, Piulats JM, Muntasell A, Dienstmann R, Lopez-Bigas N, Gonzalez-Perez A: A Pan-cancer Landscape of Interactions between Solid Tumors and Infiltrating Immune Cell Populations. Clin Cancer Res 2018, 24:3717-3728.

14. Bindea G, Mlecnik B, Tosolini M, Kirilovsky A, Waldner M, Obenauf AC, Angell H, Fredriksen T, Lafontaine L, Berger A, et al: Spatiotemporal dynamics of intratumoral immune cells reveal the immune landscape in human cancer. Immunity 2013, 39:782-795.

15. Charoentong P, Finotello F, Angelova M, Mayer C, Efremova M, Rieder D, Hackl H, Trajanoski Z: Pancancer Immunogenomic Analyses Reveal Genotype-Immunophenotype Relationships and Predictors of Response to Checkpoint Blockade. Cell Rep 2017, 18:248-262.

16. Yang XJ, Zhao JJ, Chen WJ, Zhang GG, Wang W, Tao HC: Silencing long non-coding RNA, differentiation antagonizing non-protein coding RNA promotes apoptosis and inhibits tumor growth in colon cancer. Oncol Lett 2018, 16:2865-2872.

17. Yang C, Pan Y, Deng SP: Downregulation of IncRNA CCAT1 enhances 5-fluorouracil sensitivity in human colon cancer cells. BMC Mol Cell Biol 2019, 20:9. 
18. Wu XL, Lu RY, Wang LK, Wang YY, Dai YJ, Wang CY, Yang YJ, Guo F, Xue J, Yang DD: Long noncoding RNA HOTAIR silencing inhibits invasion and proliferation of human colon cancer LoVo cells via regulating IGF2BP2. J Cell Biochem 2018.

19. Kotzin JJ, Spencer SP, McCright SJ, Kumar DBU, Collet MA, Mowel WK, Elliott EN, Uyar A, Makiya MA, Dunagin MC, et al: The long non-coding RNA Morrbid regulates Bim and short-lived myeloid cell lifespan. Nature 2016, 537:239-243.

\section{Figures}



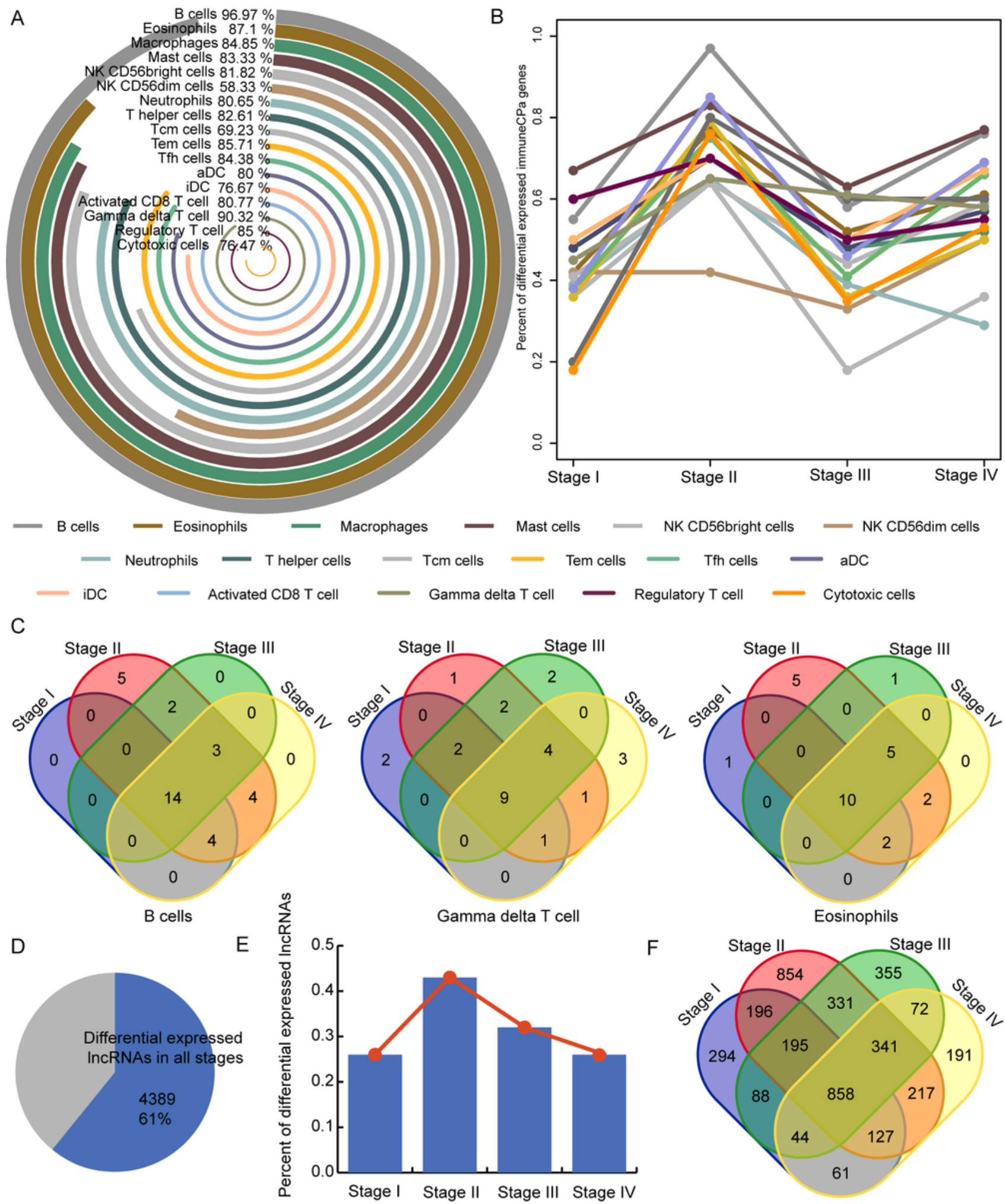

\section{Figure 1}

Stage-specific immuneCPa genes and IncRNAs are identified in colon cancer. (A) The circle diagram shows percent of differential expressed immuneCPa genes for diverse immune cell populations in all colon cancer patients. (B) Line charts show percent of differential expressed immuneCPa genes for diverse immune cell populations in diverse stages. (C) Venn diagrams show the overlap of differential expressed immuneCPa genes about B cells, Gamma delta T cell and Eosinophils in different stages. (D) 
Pie chart show percent of differential expressed IncRNAs in all colon cancer patients. (E) Percent of differential expressed immuneCPa IncRNAs for diverse immune cell populations in diverse stages. $(F)$ Venn diagrams show the overlap of differential expressed IncRNAs in different stages.

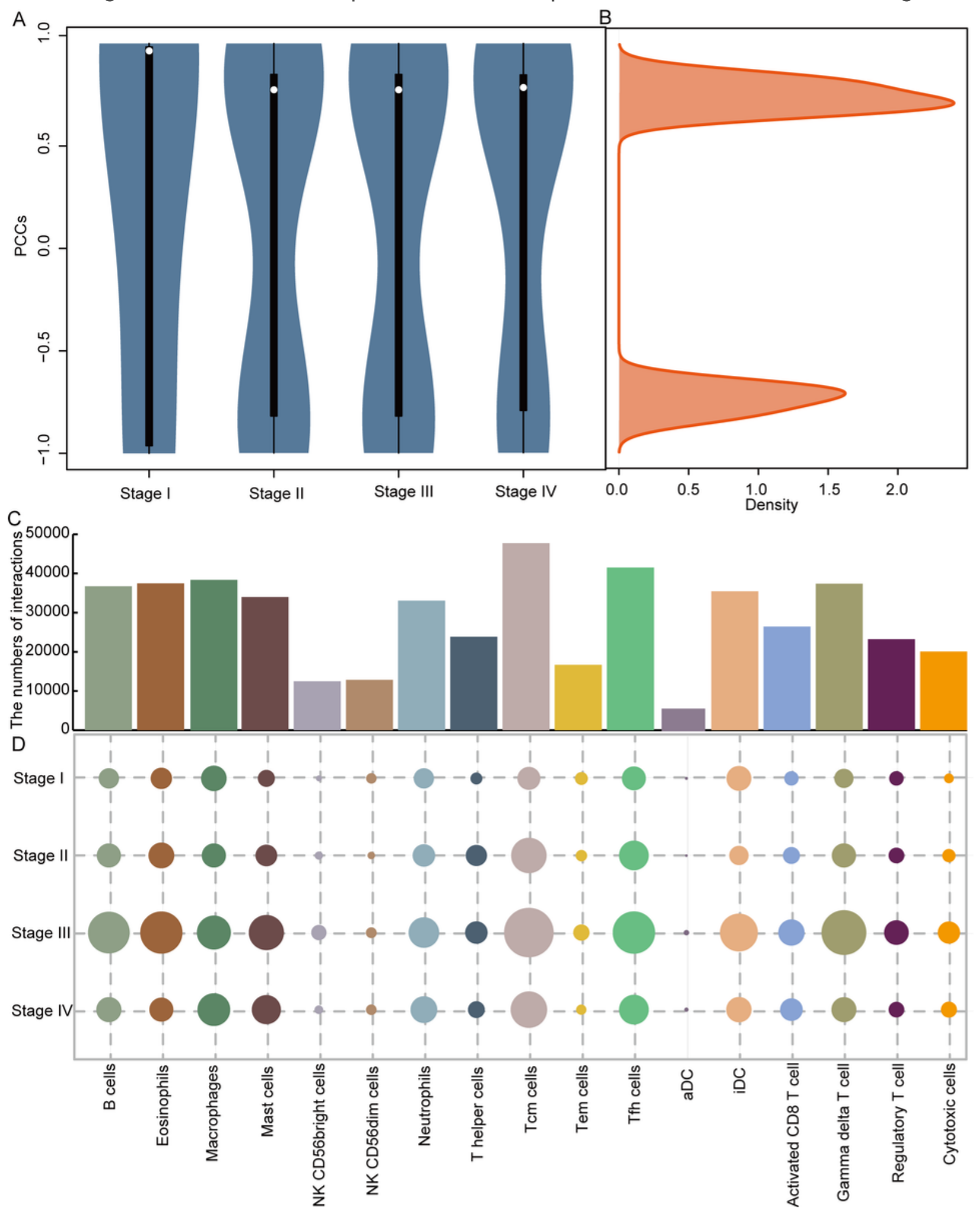

Figure 2

Identification of stage-specific immuneCPa gene-IncRNA regulatory pairs in colon cancer development. (A) Violin plots show PCCs of immuneCPa gene-IncRNA regulatory pairs in diverse stages. (B) The curve 
show density distribution for all immuneCPa gene-IncRNA regulatory pairs. (C) The bar plots show numbers of immuneCPa gene-IncRNA regulatory pairs for diverse kinds of immune cell populations. (D) The point plot show numbers of immuneCPa gene-IncRNA regulatory pairs for diverse kinds of immune cell populations in each stage.

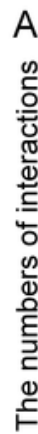

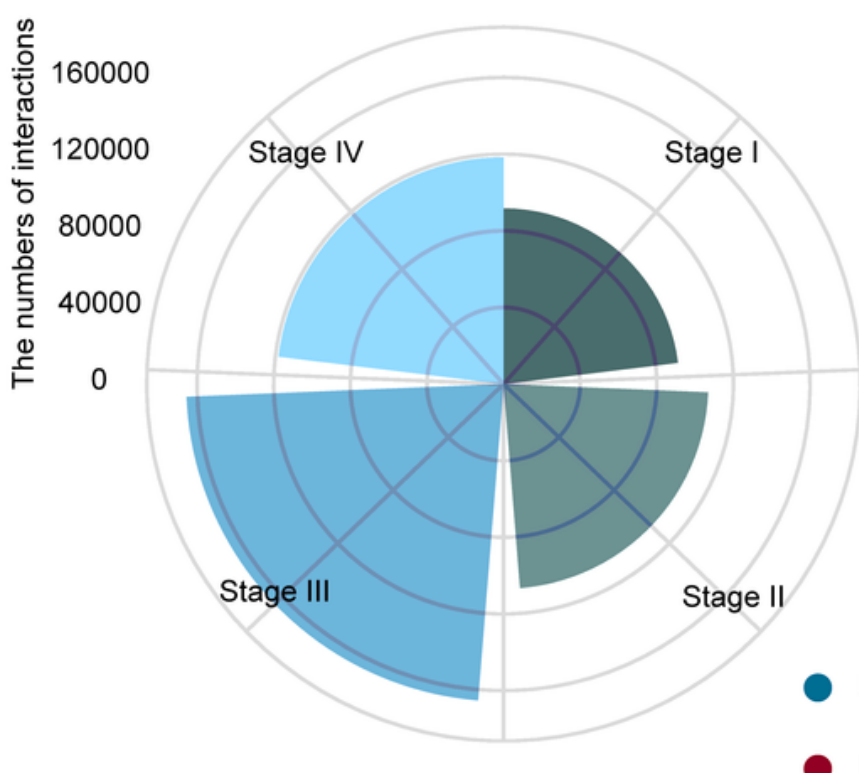

ImmuneCPa genes
B

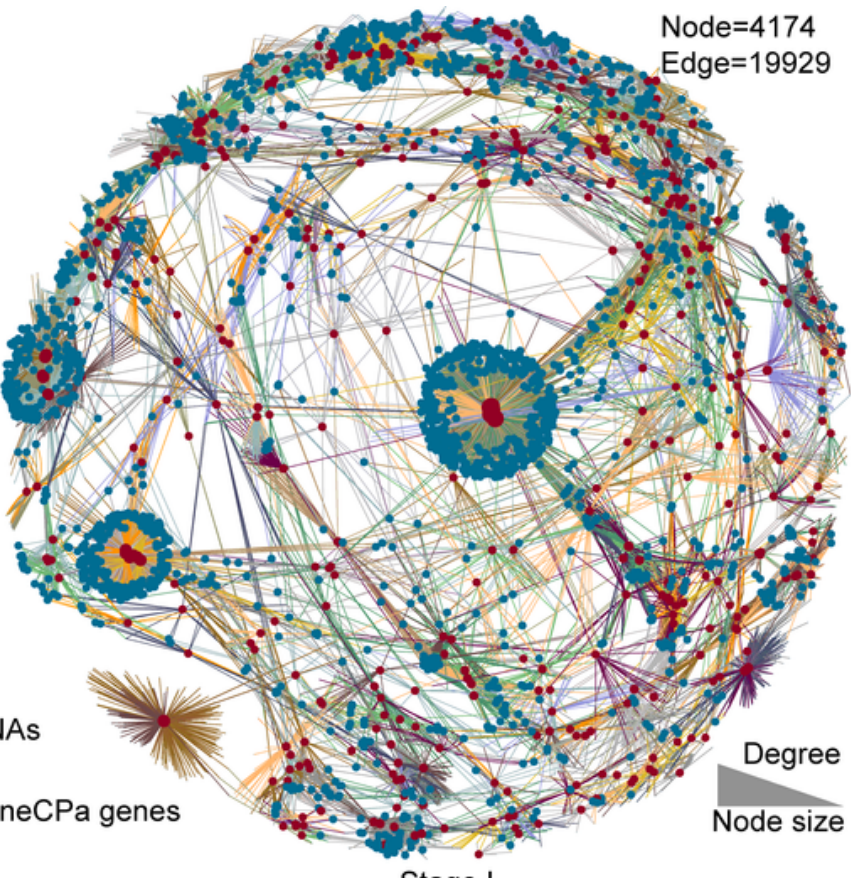

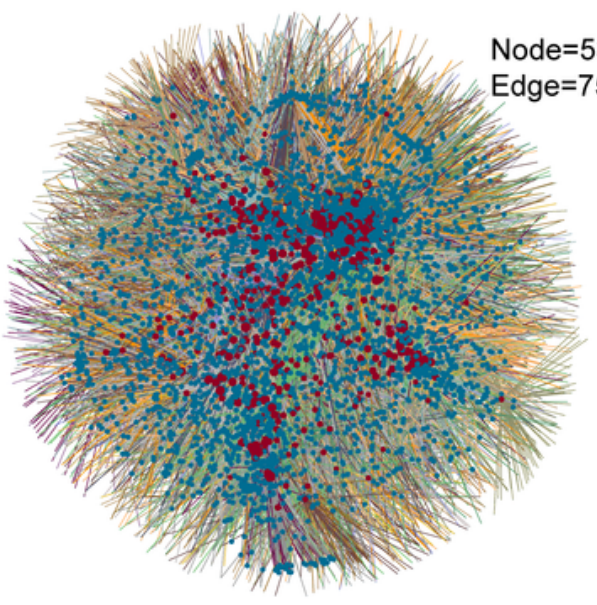

C

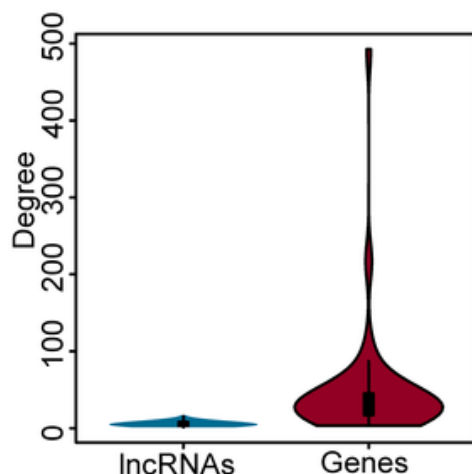

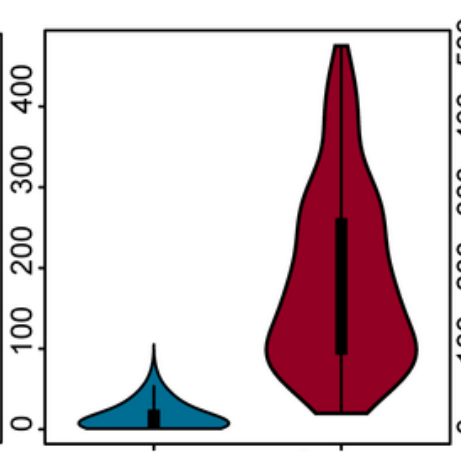

IncRNAs

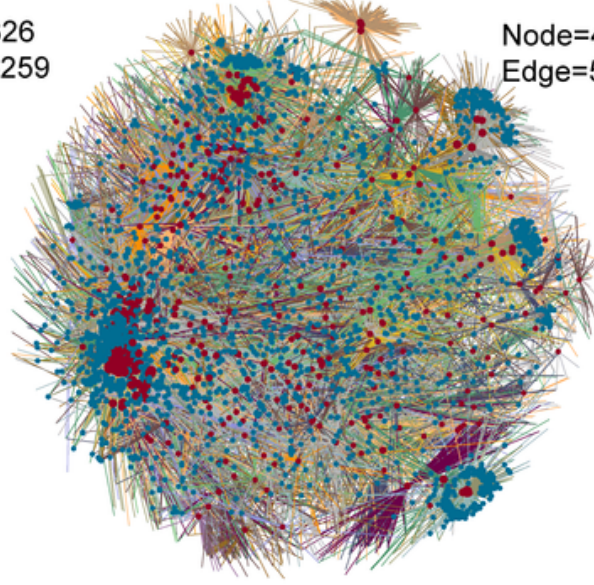

Stage III

Node $=4795$ Edge $=118356$

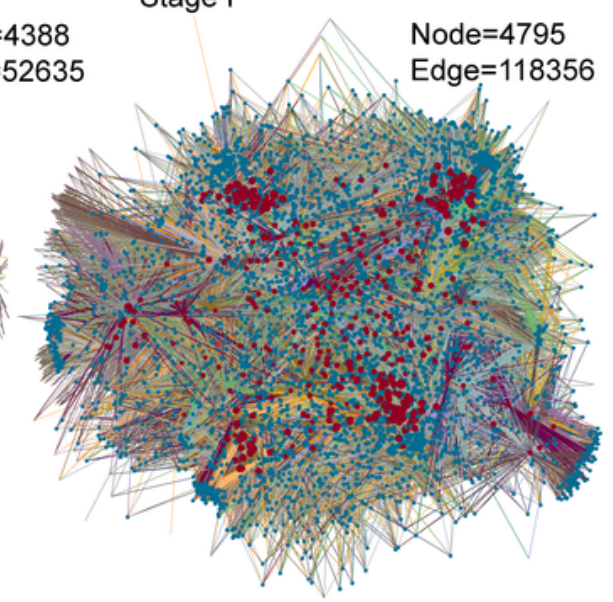

Stage IV
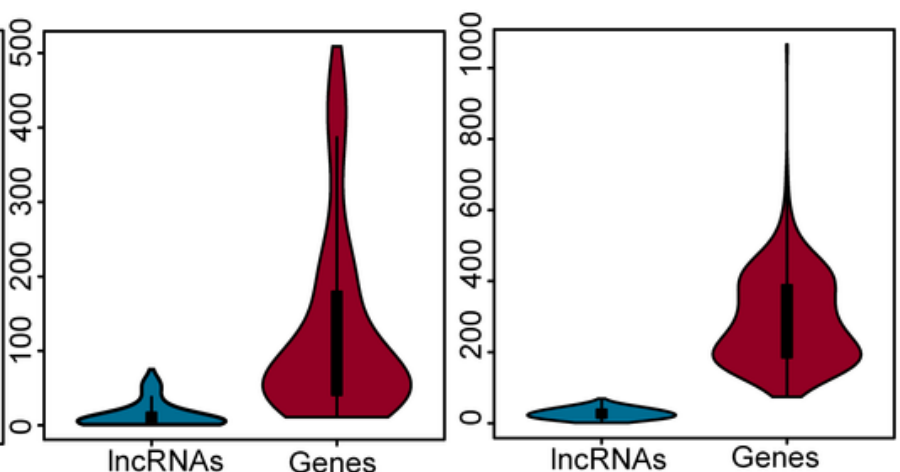

Figure 3 
Construction of dynamic stage-specific immuneCPa gene-IncRNA regulatory networks in colon cancer development. (A) The numbers of immuneCPa gene-IncRNA regulatory pairs for networks in diverse stage. (B) Network illustration of the four stage-specific immuneCPa gene-IncRNA regulatory networks. (C) Violin plots show degree of immuneCPa genes and IncRNA in four stages.
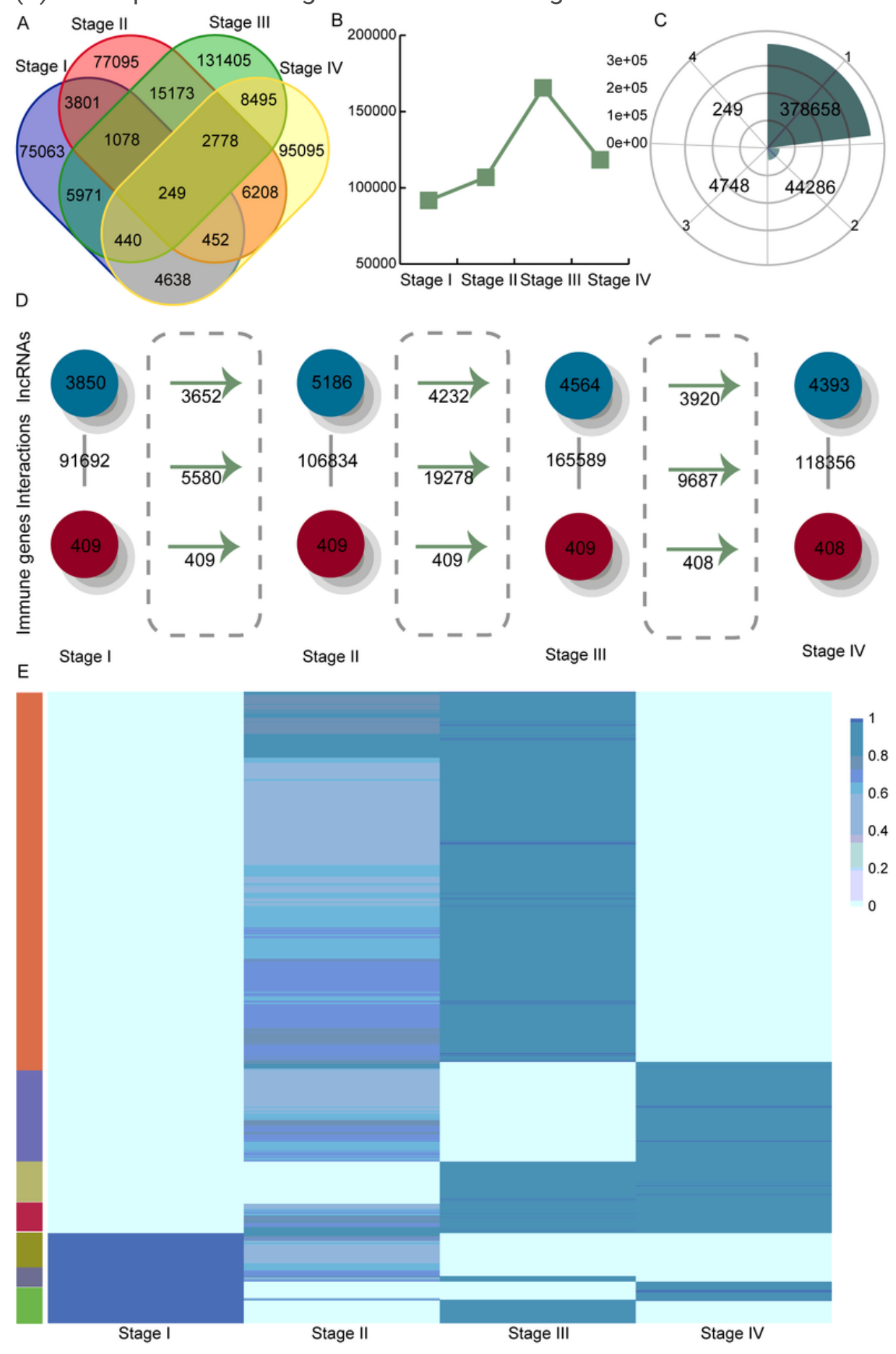

Figure 4 
The dynamic activity profiles of immuneCPa gene-IncRNA regulatory pairs. (A) Venn diagrams show the overlap of immuneCPa gene-IncRNA regulatory pairs in different stages. (B) Line chart show percent of immuneCPa gene-IncRNA regulatory pairs in diverse stages. (C) Rose diagram show the numbers of present stages for immuneCPa gene-IncRNA regulatory pairs. (D) The dynamic retaining of immuneCPa gene-IncRNA nodes and regulations across different stages of colon cancer. (E) The activity profile for immuneCPa gene-IncRNA relationships across different stages of colon cancer. The PCCs for immuneCPa gene-IncRNA pairs in each stage of colon cancer were used as activity scores. Clustering analysis revealed that different groups of immuneCPa gene-IncRNA relationships were apparently activated in one or more stages of colon cancer. 

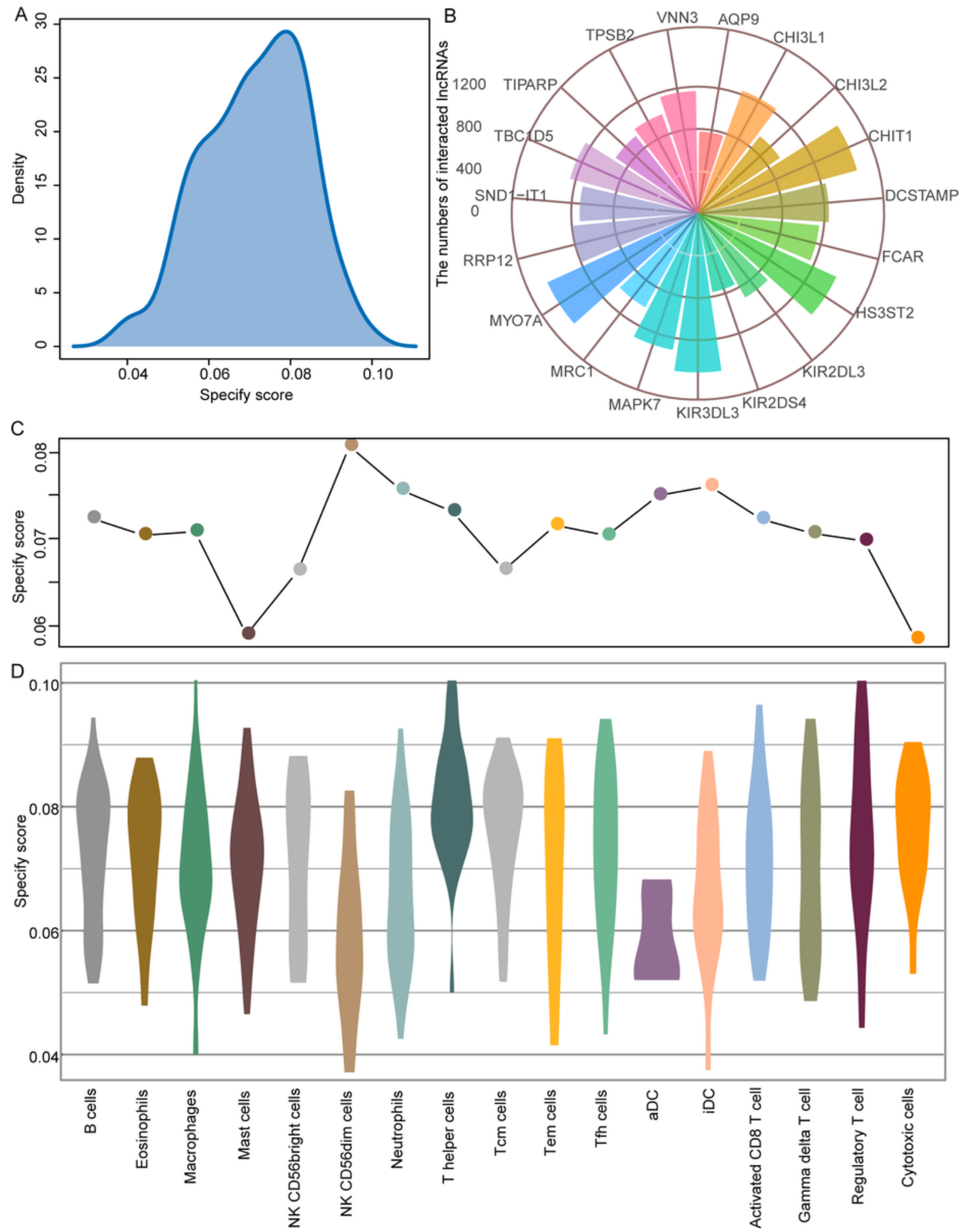

Figure 5

Specificity score evaluates the specificity of immuneCPa gene-IncRNA relationships in colon cancer development. (A) The curve shows density distribution of specificity scores of immuneCPa gene-IncRNA relationships in colon cancer. (B) The numbers of interacted IncRNAs for immuneCPa genes with lowest specificity scores. (C) Mean specificity scores of multiple kinds of immune cell populations in colon cancers. (D) Specificity scores distribution of multiple kinds of immune cell populations in colon cancers. 


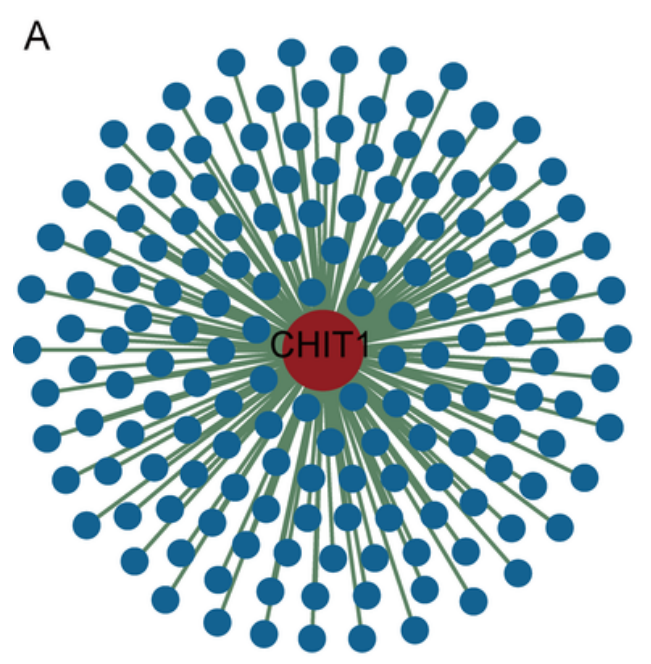

Stage II Macrophages

B
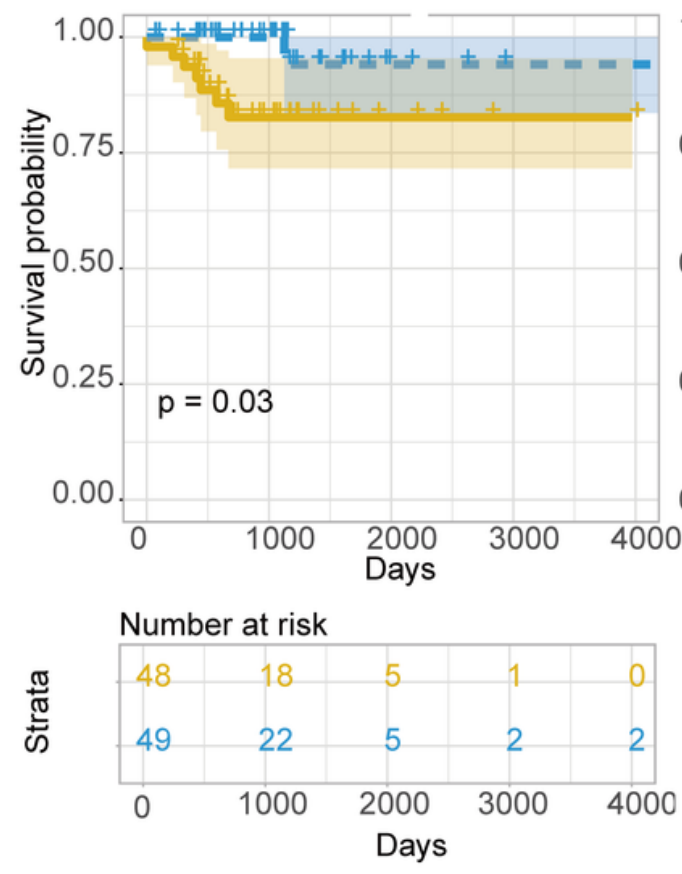

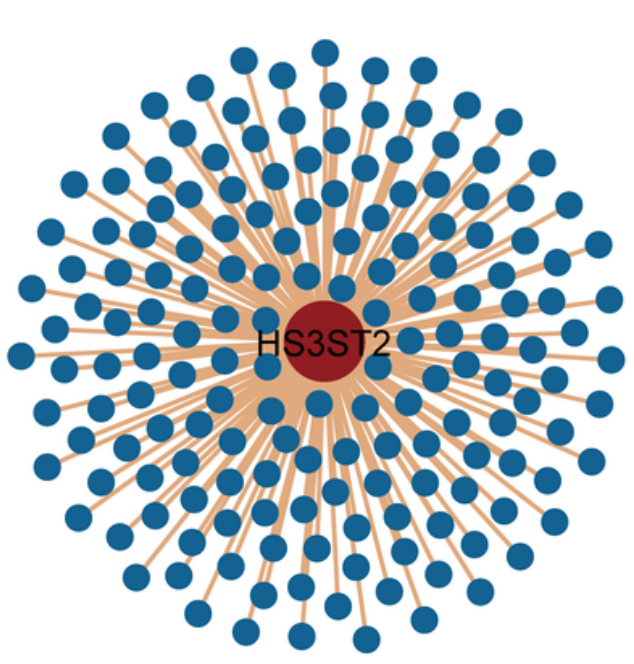

Stage II iDC

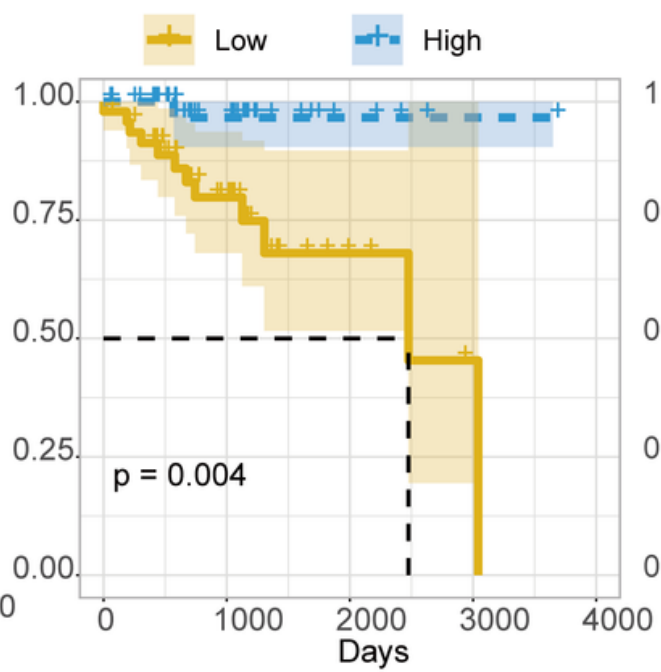

Number at risk

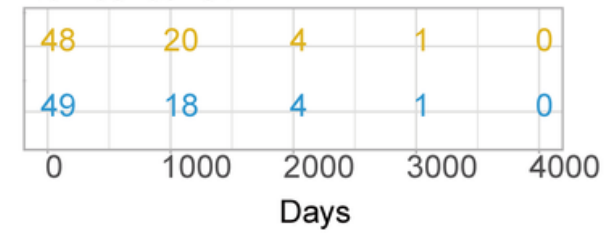

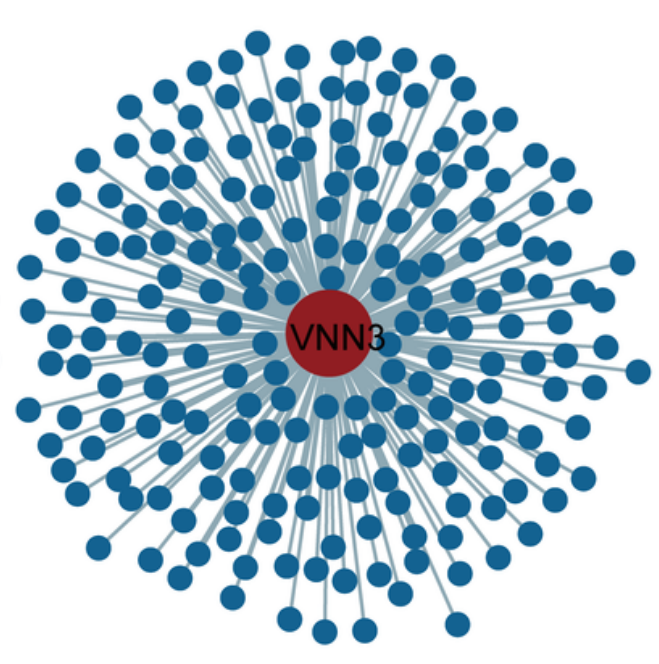

Stage III Neutrophils

Figure 6

[No caption was provided with this version.]

\section{Supplementary Files}

This is a list of supplementary files associated with this preprint. Click to download.

- Equation1.pdf 